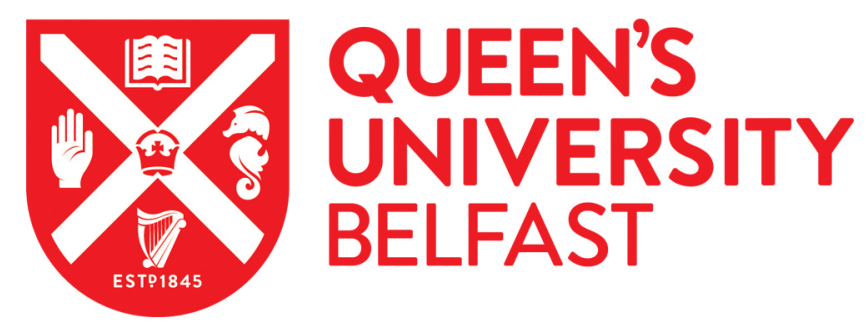

\title{
Non-equilibrium modeling of the FE XVII 3C/3D Line Ratio in an Intense X-ray Free-electron Laser Excited Plasma
}

Loch, S. D., Ballance, C. P., Li, Y., Fogle, M., \& Fontes, C. J. (2015). Non-equilibrium modeling of the FE XVII 3C/3D Line Ratio in an Intense X-ray Free-electron Laser Excited Plasma. The Astrophysical Journal Letters, 801(1), [L13]. https://doi.org/10.1088/2041-8205/801/1/L13

Published in:

The Astrophysical Journal Letters

Queen's University Belfast - Research Portal:

Link to publication record in Queen's University Belfast Research Portal

Publisher rights

(C) 2015. The American Astronomical Society. All rights reserved. Reproduced by permission of the AAS.

\section{General rights}

Copyright for the publications made accessible via the Queen's University Belfast Research Portal is retained by the author(s) and / or other copyright owners and it is a condition of accessing these publications that users recognise and abide by the legal requirements associated with these rights.

Take down policy

The Research Portal is Queen's institutional repository that provides access to Queen's research output. Every effort has been made to ensure that content in the Research Portal does not infringe any person's rights, or applicable UK laws. If you discover content in the Research Portal that you believe breaches copyright or violates any law, please contact openaccess@qub.ac.uk. 


\title{
NON-EQUILIBRIUM MODELING OF THE FE XVII 3C/3D LINE RATIO IN AN INTENSE X-RAY FREE-ELECTRON LASER EXCITED PLASMA
}

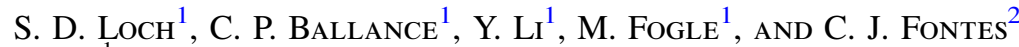 \\ ${ }^{1}$ Auburn University, Auburn, AL 36849, USA; loch@physics.auburn.edu \\ 2 Los Alamos National Laboratory, MS F663, Los Alamos, NM 87545, USA \\ Received 2014 July 23; accepted 2015 February 9; published 2015 March 2
}

\begin{abstract}
Recent measurements using an X-ray Free Electron Laser (XFEL) and an Electron Beam Ion Trap at the Linac Coherent Light Source facility highlighted large discrepancies between the observed and theoretical values for the $\mathrm{Fe}$ XVII 3C/3D line intensity ratio. This result raised the question of whether the theoretical oscillator strengths may be significantly in error, due to insufficiencies in the atomic structure calculations. We present time-dependent spectral modeling of this experiment and show that non-equilibrium effects can dramatically reduce the predicted $3 \mathrm{C} / 3 \mathrm{D}$ line intensity ratio, compared with that obtained by simply taking the ratio of oscillator strengths. Once these non-equilibrium effects are accounted for, the measured line intensity ratio can be used to determine a revised value for the $3 \mathrm{C} / 3 \mathrm{D}$ oscillator strength ratio, giving a range from 3.0 to 3.5 . We also provide a framework to narrow this range further, if more precise information about the pulse parameters can be determined. We discuss the implications of the new results for the use of Fe XVII spectral features as astrophysical diagnostics and investigate the importance of time-dependent effects in interpreting XFEL-excited plasmas.
\end{abstract}

Key words: atomic data - atomic processes - line: formation - plasmas - X-rays: general

\section{INTRODUCTION}

Fe XVII spectral emission lines have ubiquitous use in X-ray plasma spectroscopy. They are used for a range of diagnostics for both astrophysical (Hwang et al. 1992; Paerels \& Kahn 2003) and laboratory plasmas that contain Fe (Phillips et al. 1997). However, there has been some reluctance to use Fe XVII emission lines within the astrophysics community due to a number of discrepancies between observations and theory. In particular there has been a long standing discrepancy involving the ratio of the emission from the $3 \mathrm{C}\left(2 \mathrm{p}^{5} 3 \mathrm{~d}\left({ }^{1} \mathrm{P}_{1}\right) \rightarrow 2 \mathrm{p}^{6}\right.$ $\left.\left({ }^{1} \mathrm{~S}_{1}\right)\right)$ transition to the $3 \mathrm{D}\left(2 \mathrm{p}^{5} 3 \mathrm{~d}\left({ }^{3} \mathrm{D}_{1}\right) \rightarrow 2 \mathrm{p}^{6}\left({ }^{1} \mathrm{~S}_{1}\right)\right)$ transition.

A review of astrophysical and laboratory results for the $\mathrm{Fe}$ XVII 3C/3D line intensity ratio has been given by Brown (2008) and Bernitt et al. (2012). A number of important effects have been identified, such as blending with an inner shell satellite line of Fe XVI (Brown et al. 2001), cascades from higher levels, and non-Maxwellian electron distributions for EBIT experiments (Loch et al. 2006; Chen 2007; Gu 2009). Considering the EBIT measurements, the $3 \mathrm{C} / 3 \mathrm{D}$ line intensity ratio has been measured to be $3.04 \pm 0.12$ (Brown et al. 1998), $2.77 \pm 0.3$ at $910 \mathrm{eV}$ and $2.98 \pm 0.3$ at $964 \mathrm{eV}$ (Brown et al. 2006), and $\sim 2.75$ at $1100 \mathrm{eV}$ (Gillaspy et al. 2011). Theoretical modeling of these experiments produced mixed agreement. A calculation by Chen (Gillaspy et al. 2011) determined a ratio of 2.95 at $1100 \mathrm{eV}$, while the data of Loch et al. (2006) produced a ratio of 3.25. Gu (2009) also presented a comparison with EBIT measured excitation cross sections, with his modified $3 \mathrm{C}$ and $3 \mathrm{D}$ cross sections being about $15 \%$ higher than values reported by Brown et al. (2006).

The discrepancies between theory and these measurements, along with the need to include collisional-radiative effects when modeling the EBIT experiments, prompted Bernitt et al. (2012) to combine an X-ray Free Electron Laser (XFEL) at the Linac Coherent Light Source (LCLS) facility with an EBIT. Well-defined X-ray pulses scanned over the excitation energies of the associated $3 \mathrm{C}$ and $3 \mathrm{D}$ states while simultaneously measuring the emission spectrum. The expectation was that the ratio of the measured emission for each line should be a direct indication of the oscillator strength ratio (henceforward $f$-ratio), and hence to determine if inaccuracies in the $\mathrm{Fe}^{16+}$ atomic structure could be a contributing factor in the previous EBIT $3 \mathrm{C} / 3 \mathrm{D}$ discrepancies. Their measurements produced a $3 \mathrm{C} / 3 \mathrm{D}$ line intensity ratio of $2.61 \pm 0.23$, compared with calculated $f$ ratios ranging from 3.42 to 5.5 , suggesting a serious problem with the theoretical atomic structure.

The LCLS experiment and the previous EBIT experiments are quite different in nature. The EBIT experiments were electron-impact dominated plasmas, with many levels being simultaneously excited. The LCLS measurements were laserdriven and tuned to excite one transition at a time, producing a two-level system. Thus, we make a distinction between the line intensity ratio from the previous EBIT measurements and the line intensity ratio from the LCLS measurements.

The focus of this Letter is on the interpretation of the LCLS line intensity measurements and an accurate determination of the $3 \mathrm{C} / 3 \mathrm{D} f$-ratio. Radiative lifetimes for the $3 \mathrm{C}$ and $3 \mathrm{D}$ levels are 45 and $163 \mathrm{fs}$, respectively, and the XFEL produces pulse envelopes between 200 and 2000 fs (Bernitt et al. 2012). Thus, we expect non-equilibrium conditions and use time-dependent spectral modeling for the LCLS experiment.

Next we describe the details of the experiment, before building a theoretical spectral model for the emission. Our results section will initially illustrate the general role of homogeneous pulses on the $3 \mathrm{C} / 3 \mathrm{D}$ line intensity ratio before employing pulse profiles more consistent with the experimental conditions. Finally, we use the LCLS measured 3C/3D line intensity ratio to constrain the $3 \mathrm{C} / 3 \mathrm{D} f$-ratio, before presenting our conclusions.

\section{THE LCLS + EBIT EXPERIMENT}

The parameters for the XFEL pulse during the experiment of Bernitt et al. (2012) are not easily determined. However, we estimate a set of pulse parameters for the duration, intensity, 
and temporal profile based upon available literature values. The pulse durations were 200-2000 fs (Bernitt et al. 2012), however, the majority of the pulses were 200-500 fs (G.V. Brown 2014, private communication). After the soft X-ray (SXR) monochromator the energy width was $0.8 \mathrm{eV}$. The SXR web page $^{3}$ gives the number of photons after the monochromator, accounting for both filtering and optical losses. For a $500 \mathrm{eV}$ pulse, $1 \times 10^{10}$ photons are typically present and for a $1000 \mathrm{eV}$ pulse (close to the energies used in the experiment), $3 \times 10^{12}$ photons are present, giving energies per pulse of $0.0013-0.39 \mathrm{~mJ}$. The pulse focal diameters can range from 3 to $10 \mu \mathrm{m}$ (Heimann et al. 2011). The beam was weakly focused, thus we assume a focal diameter of $10 \mu \mathrm{m}$. The pulse excited one atomic transition at a time. Using these values, we obtain a radiation field density ranging from $5.77 \times 10^{-7}$ to $4.33 \times 10^{-4} \mathrm{~J} \mathrm{~m}^{-3} \mathrm{~Hz}^{-1}$. We will show that this range of values leads to a reduction in the $3 \mathrm{C} / 3 \mathrm{D}$ line intensity ratio. We also discuss the possible measured line intensity ratios if the radiation field densities were less than this range.

Due to the self-amplified stimulated emission process of an XFEL, the pulse consists of sharp and stochastic individual pulses of 1-2 fs in duration with similar sized random gaps between the spikes. The pulse interacted with a plasma cloud (50 $\mathrm{mm}$ long and $<0.5 \mathrm{~mm}$ in diameter) in the EBIT and the free electrons were removed. The pulse interacts with groundstate ions; no cascades from higher levels or collisional redistribution affecting the excited populations are possible. A two-level atomic model driven by laser excitation should describe the population dynamics.

A recent paper by Oreshkina et al. (2014) postulates that the omission of nonlinear dynamical effects, via a density matrix approach, is an alternative explanation for the decrease in the measured 3C/3D ratio of Bernitt et al. (2012). Here, we use conventional time-dependent collisional-radiative modeling to illustrate the importance of considering the pulse duration in relation to atomic lifetimes.

\section{THEORY}

Due to the precise nature of this experiment, the spectral modeling is greatly simplified. The only populating mechanism of an excited state is photo-absorption from the ground while the depopulating mechanisms are spontaneous and stimulated emission to the ground. The population density of excited state $i, N_{i}$, changes as:

$$
\begin{aligned}
\frac{d N_{i}}{d t}= & N_{1}(t) \rho\left(\omega_{o}, t\right) B_{1 \rightarrow i} \\
& -N_{i}(t)\left(A_{i \rightarrow 1}+\rho\left(\omega_{o}, t\right) B_{i \rightarrow 1}\right)
\end{aligned}
$$

where $N_{1}$ is the ground state population density. $B_{1 \rightarrow i}, B_{i \rightarrow 1}$, and $A_{i \rightarrow 1}$ are the Einstein photo-absorption, stimulated emission, and spontaneous emission coefficients. $\rho$ is the radiation field density $\left(\mathrm{J} \mathrm{m}^{-3} \mathrm{~Hz}^{-1}\right)$ (proportional to laser intensity), and $\omega_{o}$ is the transition line-center angular frequency. The temporal profile of $\rho$ will initially be modeled as a homogeneous pulse and then as a stochastic profile, more typical of the XFEL pulses.

\footnotetext{
3 https://portal.slac.stanford.edu/sites/lcls_public/Instruments/SXR/Pages/ Specifications.aspx
}

\subsection{Equilibrium Model}

In an equilibrium model for the plasma emission, the $3 \mathrm{C} / 3 \mathrm{D}$ intensity line intensity ratio is usually considered to be

$$
\frac{I_{i \rightarrow 1}^{3 \mathrm{C}}}{I_{k \rightarrow 1}^{3 \mathrm{D}}}=\frac{\iint N_{i}(x, t) A_{i \rightarrow 1} d x d t}{\iint N_{k}(x, t) A_{k \rightarrow 1} d x d t},
$$

with integrals over the line of sight of the spectrometer and the duration of the plasma emission. For a homogeneous plasma and a $\rho$ that is constant in time, this ratio reduces to the $f$-ratio. Setting $d N_{i} / d t=0$ in Equation (1) gives the following expression for the equilibrium populations

$$
N_{i}=\frac{N_{1} \rho\left(\omega_{o}\right) B_{1 \rightarrow i}}{A_{i \rightarrow 1}+\rho\left(\omega_{o}\right) B_{i \rightarrow 1}} .
$$

Note that there are two limits determined by $\rho$. For a low $\rho$, spontaneous emission dominates the denominator and

$$
N_{i}^{\text {low } \rho}=\frac{N_{1} \rho\left(\omega_{o}\right) B_{1 \rightarrow i}}{A_{i \rightarrow 1}} .
$$

For a high $\rho$, stimulated emission dominates the denominator and one obtains

$$
N_{i}^{\mathrm{high} \rho}=\frac{N_{1} B_{1 \rightarrow i}}{B_{i \rightarrow 1}}=N_{1},
$$

the expected result for a strong laser-driven excitation between two states (i.e., populations equally split between the two levels). In both limiting cases and the intermediate regime, the line intensity ratio (from Equation (2)) is very close to the $f$ ratio.

Although stimulated emission is included in our population modeling, it is not included in our intensities. It remains undetected by the LCLS spectrometer as the stimulated photons would be in the same direction as the XFEL beam. Furthermore, although 3C and 3D transitions exhibit an angular dependence in emission, the polarization effect cancels in the line intensity ratio.

The theoretical values for the $3 \mathrm{C} / 3 \mathrm{D} f$-ratio vary in the literature. A large number of atomic structure calculations, particularly those subsequently employed in electron-impact collision calculations, obtain a ratio from 3.9 to 4.5 (Bhatia \& Doschek 1992; Cornille et al. 1994; Loch et al. 2006). However, the large Many Body Perturbation Theory (MBPT) calculation of Safronova et al. (2001) produces a ratio of 3.42. This is close to the Multi-Configuration Dirac Fock (MCDF) value reported by Bernitt et al. (2012). They noted the slow convergence of the $f$-ratio, with smaller calculations producing a ratio closer to four. Gu (2009) performed a MBPT calculation giving an $f$-ratio of 3.5 . Unable to perform a full scattering calculation based upon his MBPT calculation, he applied an approximate correction to distorted-wave excitation cross sections. His modeled 3C/3D line intensity ratio was in good agreement with a range of astrophysical measurements, giving a ratio of about 3.3 at $500 \mathrm{eV}$. His modified 3C and 3D cross sections were about $15 \%$ higher than the cross sections reported by Brown et al. (2006). Chen has produced several Dirac Rmatrix calculations for $\mathrm{Fe}^{16+}$ (e.g., Chen 2007, 2008; Gillaspy et al. 2011). One of the calculations (Chen 2007) produces an $f$-ratio of 3.54 . None of the reported atomic structure calculations produce an $f$-ratio within the uncertainty of the LCLS measurements. The aim of this Letter is to explain this 


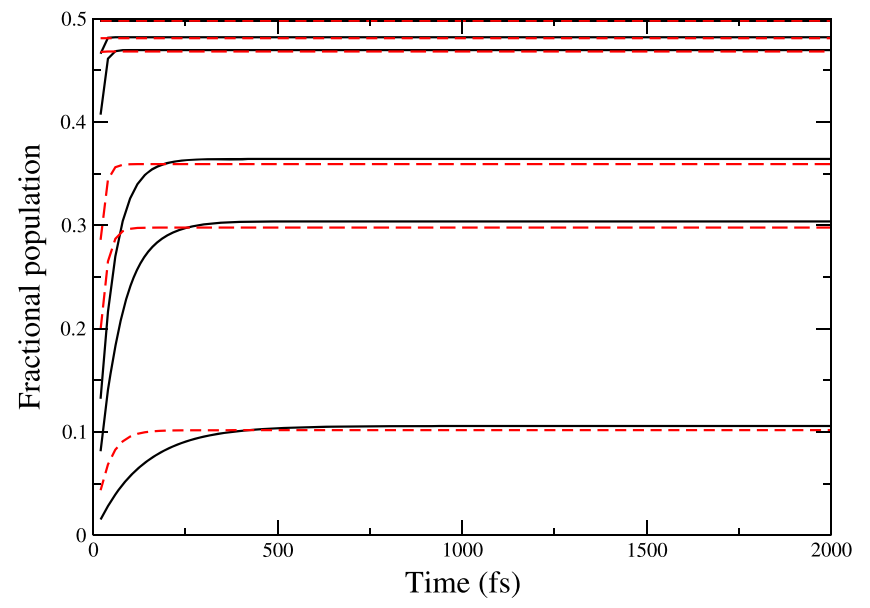

Figure 1. Fractional population in the excited state as a function of time, i.e., $N_{2} / N_{\text {tot }}$ : results for the 3D transition (solid line) and the 3C transition (dashed line). Reading from the lowest to the highest set of lines the results are for: $\rho$ $=1 \times 10^{-7}, \rho=5.77 \times 10^{-7}, 1 \times 10^{-6}, 5.77 \times 10^{-6}, 1 \times 10^{-5}$, and $1 \times 10^{-4}$ $\mathrm{J} \mathrm{m}^{-3} \mathrm{~Hz}^{-1}$.

discrepancy. We initially explore the modeling results using an $f$-ratio of 3.5 to illustrate the mechanism for the reduction in the line intensity ratio. We then invert the problem, using the LCLS measurements to put a range of values on the $f$-ratio.

\subsection{Non-equilibrium Modeling Using the Rate Equations}

Given the experimental conditions, the excitation and emission of the 3C (or 3D) spectral lines can be described by a time dependent two-level system,

$$
\begin{aligned}
\frac{d N_{1}}{d t}= & -N_{1}(t) \rho\left(\omega_{o}, t\right) B_{1 \rightarrow 2} \\
& +N_{2}(t)\left(A_{2 \rightarrow 1}+\rho\left(\omega_{o}, t\right) B_{2 \rightarrow 1}\right) \\
\frac{d N_{2}}{d t}= & N_{1}(t) \rho\left(\omega_{o}, t\right) B_{1 \rightarrow 2} \\
& -N_{2}(t)\left(A_{2 \rightarrow 1}+\rho\left(\omega_{o}, t\right) B_{2 \rightarrow 1}\right) .
\end{aligned}
$$

We assume initial conditions of $N_{1}=1$ and $N_{2}=0$, calculating the fractional population of the excited state $\left(N_{2} / N_{\text {tot }}\right)$ as a function of time $\left(N_{\text {tot }}=N_{1}+N_{2}\right)$.

Figure 1 shows the time-dependent excited populations during a homogeneous pulse. For small $\rho$ there can be significant non-equilibrium conditions at early times. For larger $\rho$, the populations reach equilibrium within a few fs and tend to the limiting value of Equation (5). The $3 \mathrm{C}$ populations reach equilibrium earlier than the 3D populations. For higher $\rho$, stimulated emission drives the populations to their steady state value quickly.

We reconstruct line intensities for each spectral line, assuming a homogeneous spatial distribution of ions, but allow the populations to evolve during the pulse. After XFEL pulse passage, no additional excitation is possible and all of the excited ions decay to the ground before the next pulse (XFEL repetition rate is $120 \mathrm{~Hz}$ ). The number of photons emitted after each pulse is given by the number of excited electrons when the pulse leaves the plasma volume. The total number of photons

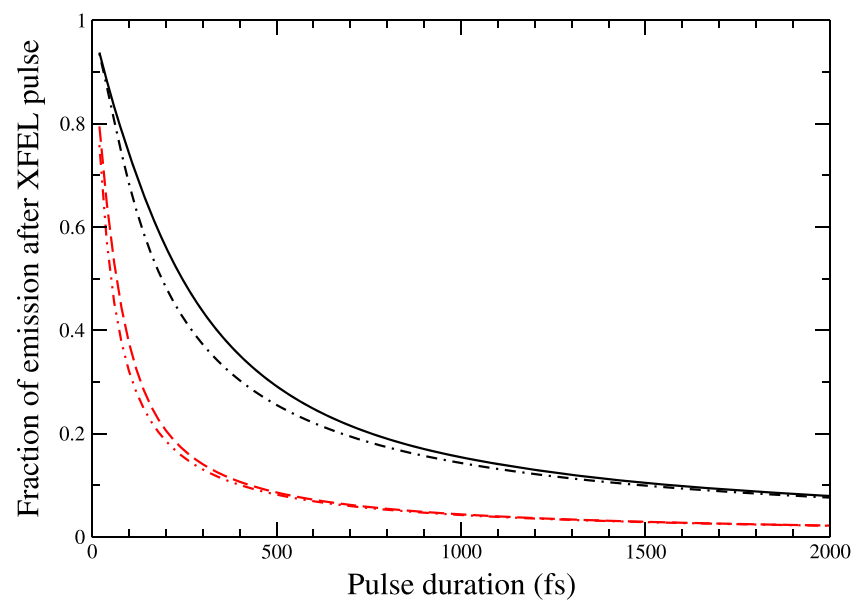

Figure 2. Fraction of the emission coming after the XFEL pulse has left the plasma volume, as a function of pulse duration for two different pulses, $\rho=5.77 \times 10^{-7}(3 \mathrm{D}:$ solid line and the 3C: dashed line) and $5.77 \times 10^{-6} \mathrm{~J} \mathrm{~m}^{-3} \mathrm{~Hz}^{-1}$ (3D: dotted-dashed line and the 3C: double-dotteddashed line).

per unit volume for a given spectral line, for a single pulse is

$$
\frac{N_{2 \rightarrow 1}^{\text {photons }}}{\Delta V}=\int_{0}^{t_{\text {pulse }}} N_{2}(t) A_{2 \rightarrow 1} d t+N_{2}\left(t_{\text {pulse }}\right) .
$$

The first term is the emission during the pulse; the second term is the emission after the pulse $\left(t_{\text {pulse }}=\right.$ pulse duration $)$. In the LCLS experiment the interaction volume overlap between the ions and the pulses $(\Delta V)$ is essentially the same, so the $\Delta V$ cancels in the $3 \mathrm{C} / 3 \mathrm{D}$ ratio. Figure 2 shows the fraction that comes after the pulse for each spectral line. The fraction of the emission after the pulse is greater for the 3D emission than for the $3 \mathrm{C}$ emission, due to the faster radiative decay of the $3 \mathrm{C}$ transition. This is a key factor in producing line intensity ratios that differ from the equilibrium values.

If the populations are driven close to their steady state value given by Equation (5), for a homogeneous pulse the line intensity ratio reduces to an analytic function of pulse duration and $A$-values.

$$
\begin{aligned}
\frac{I_{i \rightarrow 1}^{3 \mathrm{C}}}{I_{k \rightarrow 1}^{3 \mathrm{D}}} & =\frac{\int_{0}^{t_{\text {pulse }}} N_{i}(t) A_{i \rightarrow 1}^{3 \mathrm{C}} d t+N_{i}\left(t_{\text {pulse }}\right)}{\int_{0}^{t_{\text {pulse }}} N_{k}(t) A_{k \rightarrow 1}^{3 \mathrm{D}} d t+N_{k}\left(t_{\text {pulse }}\right)} \\
& =\frac{N_{i}^{\text {high } \rho} A_{i \rightarrow 1}^{3 \mathrm{C}} t_{\text {pulse }}+N_{i}^{\text {high } \rho}}{N_{k}^{\text {high } \rho} A_{k \rightarrow 1}^{3 \mathrm{D}} t_{\text {pulse }}+N_{k}^{\text {high } \rho}} \\
& =\frac{A_{i \rightarrow 1}^{3 \mathrm{C}} t_{\text {pulse }}+1}{A_{k \rightarrow 1}^{3 \mathrm{D}} t_{\text {pulse }}+1} .
\end{aligned}
$$

\section{RESULTS}

\subsection{C/3D Results for a Homogeneous XFEL Pulse}

We evaluate the 3C/3D line intensity ratio for a range of $\rho$ values and pulse durations (with $\rho$ constant over the duration of the pulse), see Figure 3. For $\rho \gtrsim 1 \times 10^{-7} \mathrm{~J} \mathrm{~m}^{-3} \mathrm{~Hz}^{-1}$, the ratio becomes smaller than the $f$-ratio. The main reason for this is the relative contribution of the emission coming during and after the pulse for the two transitions. For all of our estimated values 


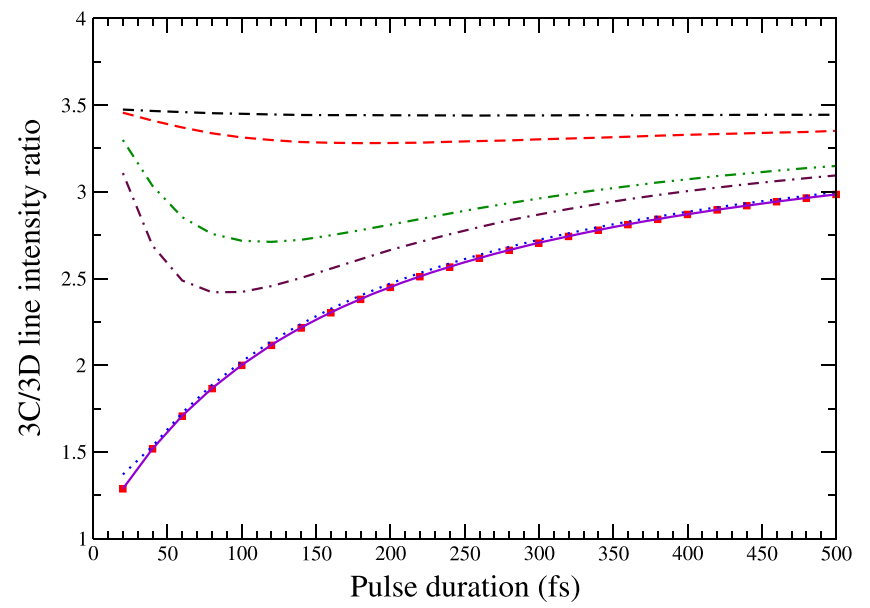

Figure 3. 3C/3D line intensity ratio as a function of pulse duration, for a range of homogeneous $\rho$ values. Results are shown for $\rho=1 \times 10^{-8}$ (double-dasheddotted line), $1 \times 10^{-7}$ (dashed line), $5.77 \times 10^{-7}$ (double-dotted-dashed line), $1.0 \times 10^{-6}$ (dotted-dashed line), $1 \times 10^{-5}$ (dotted line), and $1 \times 10^{-4}$ $\mathrm{J} \mathrm{m}^{-3} \mathrm{~Hz}^{-1}$ (solid line). The limiting value given by Equation (9) is shown (solid squares).

of $\rho$ there will be an effect on the measured line intensity ratio. For $\rho>1 \times 10^{-5} \mathrm{~J} \mathrm{~m}^{-3} \mathrm{~Hz}^{-1}$ the line intensity ratio is close to the high $\rho$ limit given by Equation (9).

Two trends are evident. As the pulse duration gets longer, more of the emission comes during the pulse and the line intensity ratio reduces to the ratio of the oscillator strengths (i.e., the first term in the numerator and denominator of Equation (9) dominates). If the pulse duration is short, most of the emission comes after the pulse (i.e., $A_{i \rightarrow 1} t_{\text {pulse }}<<1$ in Equation (9)) and the line intensity ratio tends to one. It seems likely that the range of measured values in the experiment is a reflection primarily of the sensitivity to different pulse durations.

\subsection{C/3D Results for a Stochastic XFEL Pulse}

Having indicated the mechanism for the reduction in the line intensity ratio, we now model more representative pulse profiles. A random set of Gaussian profiles ( $0.2 \mathrm{fs}$ standard deviation) were evenly distributed across the envelope, with a random number of spikes turned off. Figure 4(a) shows a typical measured profile from LCLS, and Figure 4(b) shows our stochastically generated pulse.

Figure 5 shows results for two stochastic values of $\rho$. The line intensity ratios oscillate close to the ratio for a homogeneous pulse of the same $\rho$. For most of our radiation field densities, the populations are driven to their equilibrium values well before $200 \mathrm{fs}$. In the 1-2 fs between intensity spikes, the $3 \mathrm{C}$ and $3 \mathrm{D}$ populations are only slightly reduced. Thus, the ratio stays close to that predicted from the homogeneous pulse. The gaps in the pulse allow the ratio to drop slightly below the results for the homogeneous pulse, and even below the theoretical high $\rho$ limit of Equation (9), which was derived assuming a homogeneous $\rho$.

The LCLS results were generated from the combination of a large number of individual pulses. Thus, we also evaluate a line intensity ratio based on a range of pulse durations and radiation field densities, using a set of stochastic pulses with average $\rho$ from $5.77 \times 10^{-7}$ to $4.33 \times 10^{-4} \mathrm{~J} \mathrm{~m}^{-3} \mathrm{~Hz}^{-1}$, and durations from
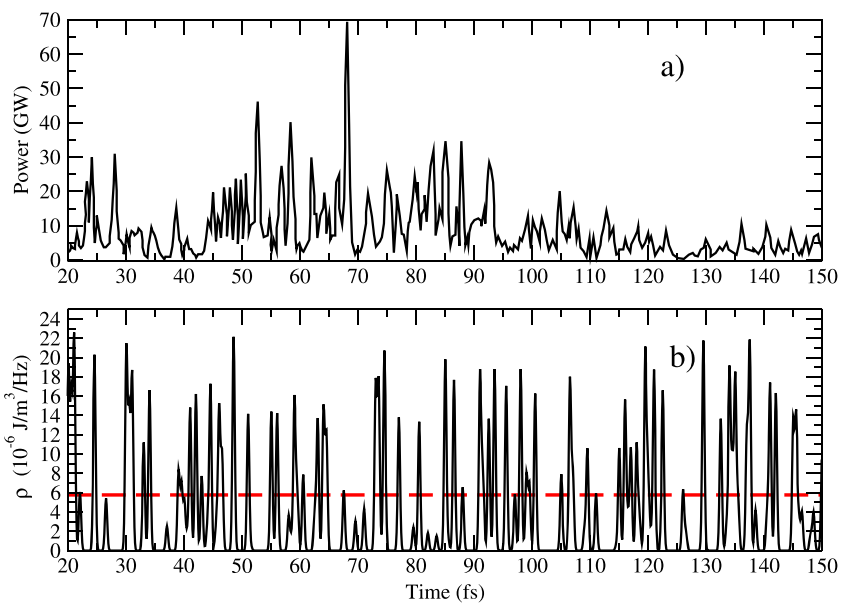

Figure 4. Figure (a) shows a typical experimentally measured XFEL profile for an $800 \mathrm{eV}$ pulse. Figure (b) shows one of our simulated stochastic pulses as a function of time for an average $\rho$ of $5.77 \times 10^{-6} \mathrm{~J} \mathrm{~m}^{-3} \mathrm{~Hz}^{-1}$. The dashed line shows a homogeneous $\rho$ of $5.77 \times 10^{-6} \mathrm{~J} \mathrm{~m}^{-3} \mathrm{~Hz}^{-1}$. The stochastic pattern continues for the duration of the pulse.

200-500 fs. We consider a normal distribution of pulse durations centered at $350 \mathrm{fs}$ with a standard deviation of 50 fs (to ensure that the majority are within 200-500 fs), and we calculate the average $3 \mathrm{C}$ and $3 \mathrm{D}$ intensities, along with their standard deviations. This gives a $3 \mathrm{C} / 3 \mathrm{D}$ ratio of $2.80 \pm 0.33$, significantly lower than the $f$-ratio of 3.5 used in the model. Thus, it appears that a large part of the discrepancy between the measured $3 \mathrm{C} / 3 \mathrm{D}$ line intensity ratio and the theoretical $f$-ratio can be explained by the non-equilibrium nature of the plasma excitation and emission.

\subsection{Using the LCLS Measurements to Determine the 3C/3D Oscillator Strength Ratio}

Given the LCLS measured line intensity ratio of $2.61 \pm 0.23$, we can use our model to draw some conclusions about the $f$ ratio. We explored the line intensity ratio results for oscillator strengths from 2.4 to 3.5 , while also considering the estimated range of pulse intensities and durations. We can make the following conclusions: if the $f$-ratio were 3.5 , the predicted intensity ratios are at the upper end of the experimental error bars, requiring the distribution of pulses to be weighted toward $200 \mathrm{fs}$ to produce ratios that are consistently within the error bars. A ratio of 3.2 is in better agreement with the experimental values if the pulses were evenly distributed within the range of estimated pulse durations. An $f$-ratio of 3.0 produces line intensity ratios that are at the lower end of the error bars and would require pulse durations that were weighted toward $500 \mathrm{fs}$ to reproduce experimental measurements. Based upon our estimated range of $\rho$ values, we expect an $f$-ratio between 3.0 and 3.5 .

If the radiation field density was lower than our estimated range, the reduction of the line intensity ratio becomes smaller and could also produce the values measured by the experiment. If the $f$-ratio were as low as 2.6 , one would require radiation field densities below $1 \times 10^{-8} \mathrm{~J} \mathrm{~m}^{-3} \mathrm{~Hz}^{-1}$, and would produce line intensity ratios at the lower end of the measured values. If the $f$-ratio were 3.0 and the radiation field density were $\sim 2 \times 10^{-7} \mathrm{~J} \mathrm{~m}^{-3} \mathrm{~Hz}^{-1}$, one would obtain line intensity ratios within the experimental error bars. 


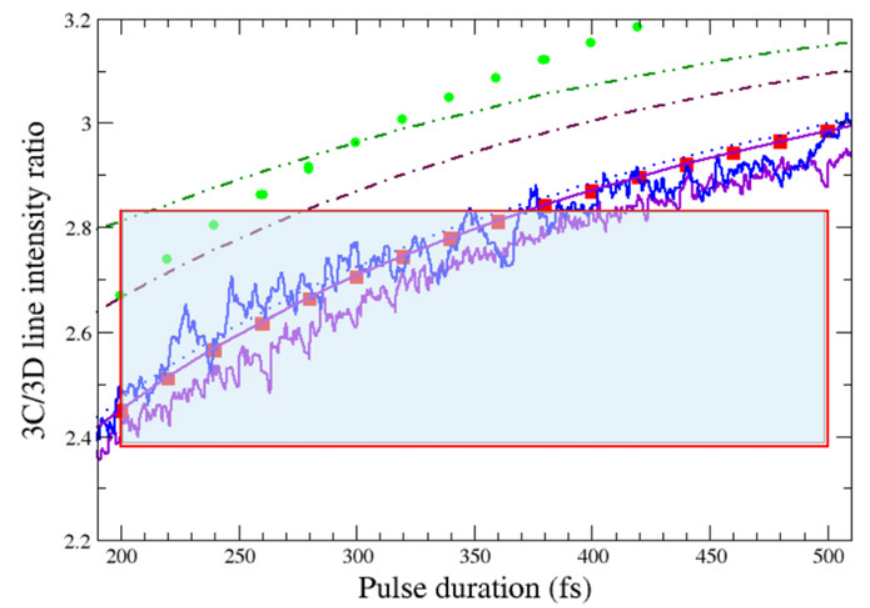

Figure 5. 3C/3D line intensity ratio as a function of pulse duration. The line styles are the same as in Figure 3. Results for stochastic pulses are show for $\rho=1.0 \times 10^{-4} \mathrm{~J} \mathrm{~m}^{-3} \mathrm{~Hz}^{-1}$ (lowest jagged line), and $\rho=1.0 \times 10^{-5} \mathrm{~J} \mathrm{~m}^{-3} \mathrm{~Hz}^{-1}$ (next higher jagged line). The squares and circles show the results using Equation (9) for an $f$-ratio of 3.5 and 3.9, respectively. The box indicates the dominant range of pulse durations and the measured ratio values.

\section{CONCLUSIONS}

We have shown that the measured $\mathrm{Fe}$ XVII $3 \mathrm{C} / 3 \mathrm{D}$ line intensity ratio of Bernitt et al. (2012) can be explained by the non-equilibrium conditions of the plasma. Once these conditions have been accounted for, the measurements can be used to deduce a new estimate for the $3 \mathrm{C} / 3 \mathrm{D}$ oscillator strength ratio. Values of 3.0-3.5 produce results within the experimental error bars, based upon an estimated set of parameters for the radiation field density.

While the focus of this Letter is on the LCLS measurements and the $3 \mathrm{C} / 3 \mathrm{D}$ oscillator strength ratio, it is useful to consider the previous EBIT measurements. Many of the previous scattering calculations that have been used to generate electron-impact data for $\mathrm{Fe}^{16+}$ spectral modeling are based upon structure calculations that produce oscillator strength ratios greater than 3.5 . One possibility is that the sensitivity to the atomic structure as revealed in oscillator strength studies (Gu 2009) also causes differences in the collision cross sections. Future collision calculations for $\mathrm{Fe}^{16+}$ should be guided by this new estimate for the oscillator strength ratio, providing an avenue to investigate the previous EBIT 3C/3D line intensity measurements. This is beyond the scope of this Letter, but will form the basis for future work.

The wider implication of this work is on the new field of XFEL driven plasma emission; account must be taken of the time-dependent effects in the plasma emission. We note that emission from higher charge states of $\mathrm{Fe}$ (such as $\mathrm{Fe}^{24+}$ ) would produce only small non-equilibrium effects and the line intensity ratio should be close to the oscillator strength ratio.

We would like to thank Greg Brown at LLNL for providing specific experimental parameters of LCLS.

\section{REFERENCES}

Bhatia, A. K., \& Doschek, G. A. 1992, ADNDT, 52, 1

Bernitt, S., Brown, G. V., Rudolph, J. K., et al. 2012, Natur, 492, 225

Brown, G. V., Beiersdorfer, P., Liedahl, D. A., Widman, K., \& Kahn, S. M. 1998, ApJ, 502, 1015

Brown, G. V., Beiersdorfer, P., Chen, H., Chen, M. H., \& Reed, K. J. 2001, ApJL, 557, L75

Brown, G. V., Beiersdorfer, P., Chen, H., et al. 2006, PhRvL, 96, 253201

Brown, G. V. 2008, CaJPh, 86, 199

Chen, G.-X. 2007, PhRvA, 76, 062708

Chen, G.-X. 2008, MNRAS, 386, L62

Cornille, M., Dubau, J., \& Jacquemot, S. 1994, ADNDT, 58, 1

Gillaspy, J. D., Lin, T., Tedesco, L., et al. 2011, ApJ, 728, 132

Gu, M. F. 2009, arXiv:0905.0519v1

Heimann, P., Krupin, O., Schlotter, W. F., et al. 2011, RScI, 82, 093104

Hwang, U., Canizares, C. R., Markert, T. H., \& Hughes, J. P. 1992, BAAS, 24, 790

Loch, S. D., Pindzola, M. S., Ballance, C. P., \& Griffin, D. C. 2006, JPhB, 39,85

Oreshkina, N. S., Cavaletto, S. M., Keitel, C. H., \& Harman, Z. 2014, PhRvL, 113, 143001

Paerels, F. B. S., \& Kahn, S. M. 2003, ARA\&A, 41, 291

Phillips, K. J. H., Greer, C. J., Bhatia, A. K., et al. 1997, A\&A, 324, 381

Safronova, U. I., Namba, C., Murakami, I., Johnson, W. R., \& Safronova, M. S. 2001, PhRvA, 64, 012507 